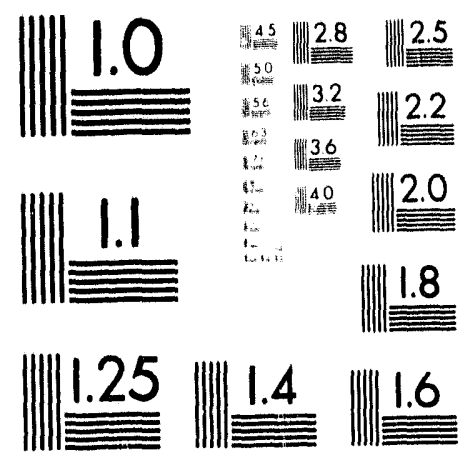



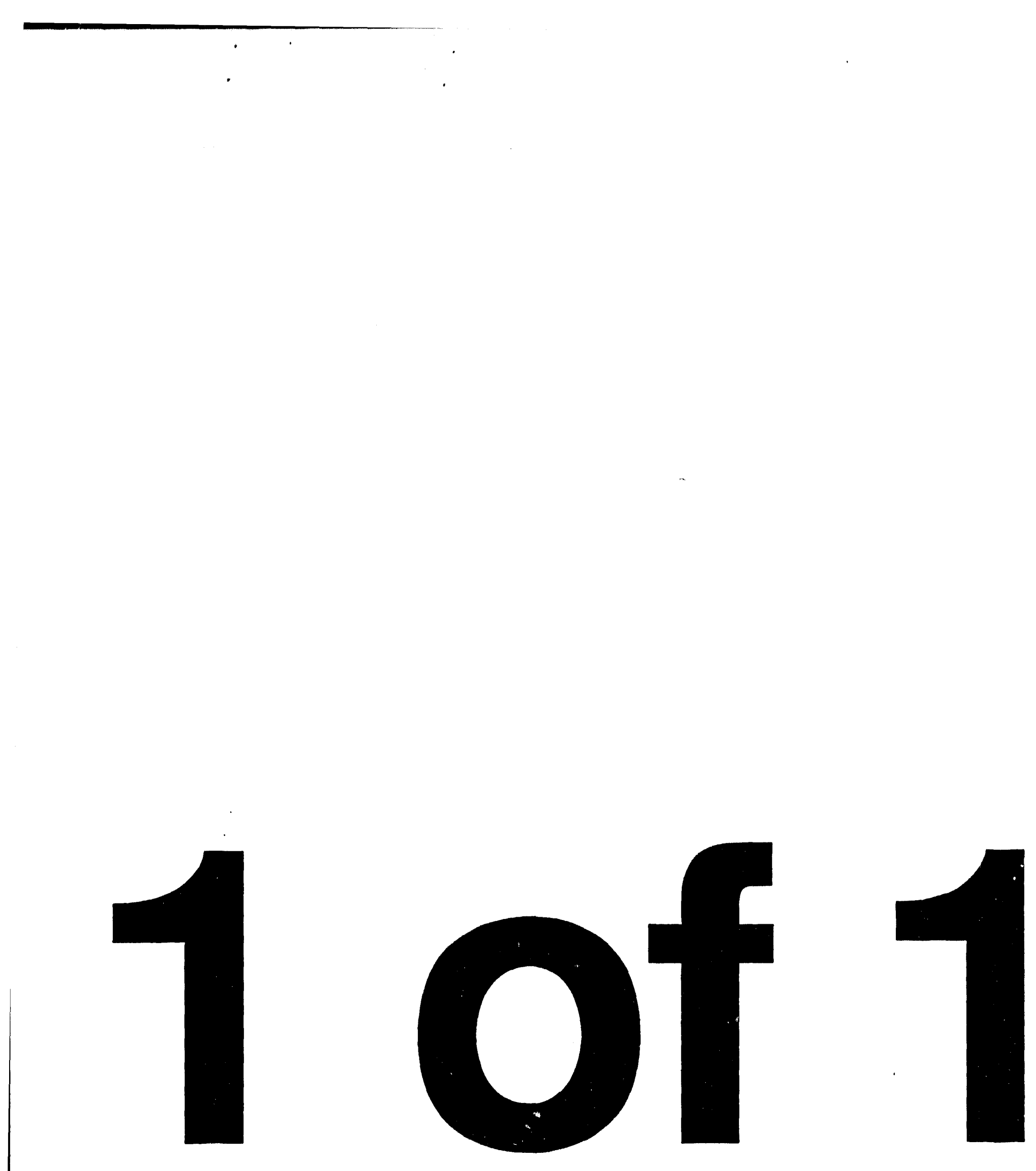


\title{
Use of the Holifield Facility 25-MV Tandem Accelerator in the Oak Ridge Radioactive Ion Beam Project*
}

\author{
C. M. Jones, R. C. Juras, M. J. Meigs, and D. K. Olsen \\ Oak Ridge National Laboratory \\ P. O. Box 2008, Oak Ridge, TN 37831-6368
}

Abstract

The absence of time structure and the excellent beam and beam transport properties of tandem electrostatic accelerators make them an attractive choice for the acceleration of a large class of radioactive ion species produced with the ISOL technique. In this paper, considerations on the use of the Holifield facility $25-\mathrm{MV}$ tandem accelerator in the ORNL Radioactive Ion Beam Facility now under construction will be presented. Preliminary beam parameter measurements which suggest that the 25 MV tandem accelerator can be used as an effective separator of isobaric contaminant beams will also be presented and discussed.

\section{INTRODUCTION}

The ORNL project $[1,2]$ to produce mediumintensity, proton-rich, radioactive ion beams (RIBs) for astrophysics, nuclear physics, and applied research with the Holifield Heavy Ion Research Facility (HHIRF) accelerators is now in progress. Radioactive atoms will be produced by fusion reactions in an Isotope Separator On-Line (ISOL)type target-ion source assembly using intense light ion beams from the Oak Ridge Isochronous Cyclotron. The radioactive atoms will be converted to negative ions ejther directly in one of several types of negative ion sources or by charge exchange following positive ionization. After acceleration to approximately $300 \mathrm{keV}$ from a high-voltage platform, these negative ions will be injected into the HHIRF 25-MV tandem accelerator [3-5] for acceleration to higher energies. Beams up to mass 76 can be accelerated to energies greater than $5 \mathrm{MeV} /$ nucleon. For some raciioactive beams, intensities greater than 1 pnA are possible.

While use of the existing HHIRF tandem accelerator was clearly dictated by its availability, it is also clear that tandem accelerators possess a number of attributes which make them an attractive choice for this type of application. The most important of these are a large phase space acceptance for the injected beam, good transmission efficiency, the absence of time structure, simplicity and reliability, the ability to accelerate singly charged injected beams with low initial velocity, and excellent accelerated beam quality. The latter property is

\footnotetext{
*Research sponsored by the U.S. Department of Energy under contact No. DE-AC05-84OR21400 with Martin Marietta Energy Systems, Inc.
}

especially important because of its implications in regard to the use of radioactive beams in the research program and because high-quality accelerated beams create the potential for using the accelerator's energyanalyzing magnet as an isobar separator. The potential disadvantage of the tandem accelerator is the requirement to produce and inject beams of negative ions. In the remainder of this paper, some of these attributes as they apply to the HHIRF tandem accelerator will be discussed along with some more general considerations on the use of tandem accelerators.

\section{INJECTED BEAM REQUIREMENTS}

Two important injected beam parameters are minimum energy and maximum emittance. In the case of the HHIRF tandem accelerator, both of these parameters are strongly influenced by the fact that the tandem accelerator is equipped with a quadrupole lens in the low-energy acceleration tube at a position which is approximately one-third of the distance between the tube entrance and exit. With the use of this lens, the low-energy acceleration tube normalized acceptance is insensitive to injection energy if the injection system can provide a beam waist at the entrance of the low-energy acceleration tube which is approximately equal in diameter to the beam waist at the terminal stripper [6]. In practice, this condition cannot be satisfied over a wide range of injection energies because only one lens was provided between the image waist of the mass-analyzing magnet and the entrance of the low-energy acceleration tube [7]. This lens was positioned to provide good matching for beams of approximately $300 \mathrm{keV}$ and cannot be easily moved because of its location with respect to the pressure vessel boundary.

The effect of lower injection energies was investigated by a series of measurements of overall optimized transmission efficiency for seven beams, typical of those expected for the RIB facility, at injection energies of 100,200, and $300 \mathrm{keV}$. The essenial result of these measurements is that transmission efficiency was reduced at $100 \mathrm{keV}$, but not at $200 \mathrm{keV}$, indicating that the tandem injection energy can be lowered to $200 \mathrm{keV}$ without a significant effect on transmission efficiency.

Using the techniques discussed by Larson and Jones [6], it is possible to estimate the phase space authored by a contractor of the U.S. Government under contract No. DE. ACO5-8AOR2 1400. Accordingly, the U.S. Government retains a nonexclusive. rovalty-free ikense to publish or reproduce the published form of this contribistion. or allow others to do so. tor U.S. Government purposes:

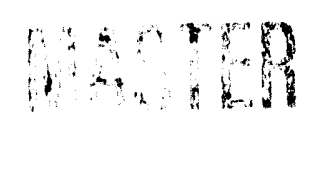


acceptance of the low-energy acceleration tube as a function of terminal potential. With the assumption of a 9.5-mm-diameter terminal stripper and operation of the full, unshorted column, the calculated normalized phase space acceptance varies between $4.1 \pi \mathrm{mm}$ mrad $\mathrm{MeV}^{1 / 2}$ at a terminal potential of $1 \mathrm{MV}$ and $17.6 \pi \mathrm{mm} \operatorname{mrad} \mathrm{MeV}^{1 / 2}$ at a terminal potential of $25 \mathrm{MV}$. These values of acceptance may be compared to the typical value of $8 \pi \mathrm{mm}$ mrad $\mathrm{MeV}^{1 / 2}(80 \%$ fraction) measured for sources now being used with the tandem accelerator [8] and to the value of approximately $2 \pi \mathrm{mm} \operatorname{mrad~} \mathrm{MeV}^{1 / 2}$ estimated for the ISOLDE-type source planned for initial use with the RIB facility [9].

It should be noted that operation of the tandem accelerator with an unshorted column at low terminal potentials will require installation of a resistor-based voltage grading system [10]. It should also be noted that a new terminal potential stahilizer system developed at ORNL allows stable operation of the tandem accelerator using only a generating voltmeter to monitor the terminal potential. Since, in this mode, the beam is not used for terminal potential stabilization, there is no minimum injected beam intensity requirement for the tandem accelerator.

\section{ACCELERATED BEAM ENERGY AND BEAM TRANSMISSION EFFICIENCY}

Beam transmission efficiency for the tandem accelerator is the product of two factors: The first factor is a beam loss factor which describes transmission losses. This factor, which has been empirically determined from operating experience, depends on ion mass and the number and type of strippers, but is approximately independent of charge state. The second factor is the product of charge state fractions in the various strippers which may be employed.

For use as a RIB accelerator, it is assumed that (1) the tandem accelerator will be used only with a terminal stripper, (2) charge state distribution functions are accurately predicted by the semiempirical parameterization of Sayer [11], and (3) use of a planned resistor-based voltage grading system [10] will enable operation at terminal potentials up to $26 \mathrm{MV}$. Maximum accelerated beam energies and corresponding transmission efficiencies, estimated with these assumptions, are shown in Fig. 1. With terminal gas stripping, beams of mass 52 can be accelerated to $5 \mathrm{MeV} / \mathrm{amu}$ with a total efficiency of $20 \%$. With terminal foil stripping, beams of mass 76 can be accelerated to $5 \mathrm{MeV} / \mathrm{amu}$ with a total efficiency of $8 \%$. In both cases, beams of lower masses can be accelerated to higher energies with total efficiencies approaching $50 \%$.

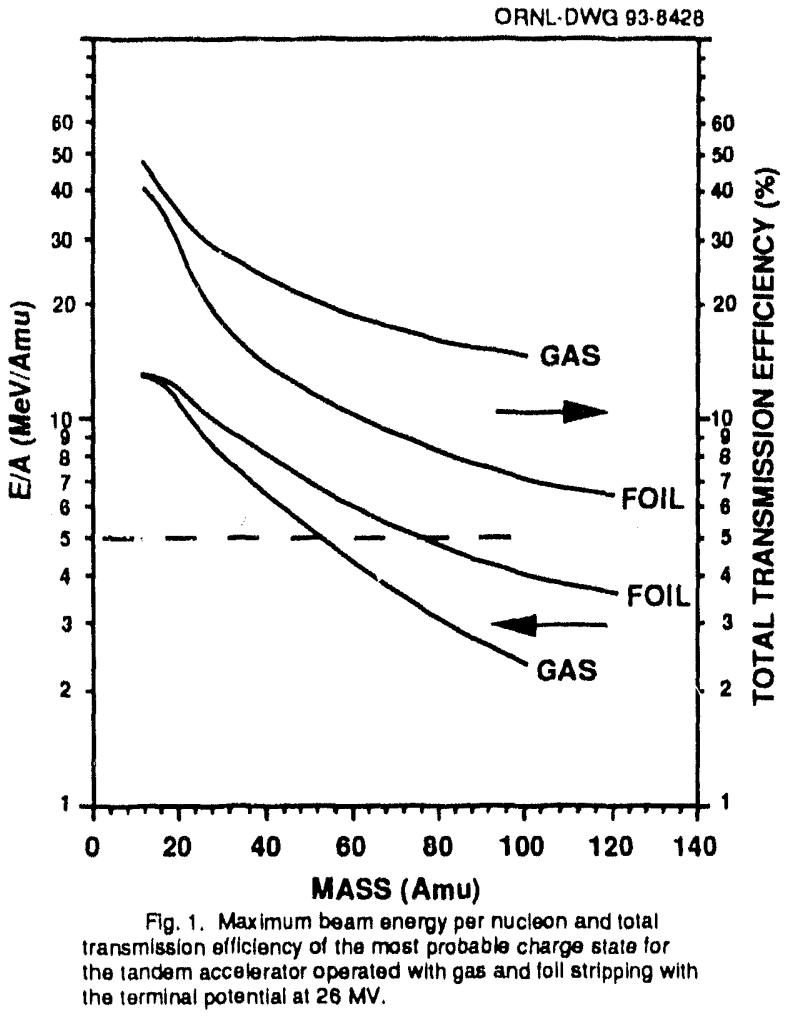

\section{ACCELERATED BEAM EMITTANCE AND USE OF THE ENERGY-ANALYZING MAGNET AS AN ISOBAR SEPARATOR}

The high quality of tandem accelerator beams suggests the possibility of using the energy-analyzing magnet as an isobar separator. In the simplest mode, the accelerator would be operated using a generating voltmeter for terminal potential stabilization and ions of differing mass would be separated at the image plane of the energy-anaiyzing magnet, which, in the case of the HHIRF tandem accelerator, is a $90^{\circ}$ double-focusing, $n=0$ magnet with mass and energy dispersion $=3.35 \mathrm{~m}$. In detail, there are several questions to be addressed: (1) What is the intrinsic energy spread of the beam; (2) What is the energy stability of the beam when opcrating with generating voltmeter stabilization; and (3) What is the emittance of the accelerated beam.

To address these questions, a series of measurements were made with ${ }^{16} \mathrm{O},{ }^{32} \mathrm{~S}$, and ${ }^{58} \mathrm{Ni}$ beams at energies which are typical of those expected for the new RIB facility. The essential results of these measurements may be summarized as follows:

- The apparent collective beam motion in the image-bending plane has a maximum amplitude of about $0.5 \mathrm{~mm}$ peak-to-peak with a dominant frequency of $2.3 \mathrm{~Hz}$. This apparent collective motion appears to have at least two components: 
1. A component which appears only in the image-bending plane and is thought to be due to tandem terminal voltage fluctuations.

2. A component which appears in both the object and image planes and is thought to be either an artifact of the measurement technique or real beam motion resulting from ion-optic component power-supply fluctuations.

Contributions of this apparent motion to beam size measurements were reduced by an averaging technique.

- Beam emittance for a $90 \%$ beam fraction is typically $0.3-0.5 \pi \mathrm{mm}$ mrad for gas stripping and $1.0 \pi \mathrm{mm}$ mrad for foil stripping.

- Bcam widths for a $90 \%$ beam fraction in the image-bending plane are typically 0.5 to $1.0 \mathrm{~mm}$ for gas stripping and $1.4 \mathrm{~mm}$ for foil stripping.

- With the addition of a quadrupole lens upstream of the energy-analyzing magnet, beam widths for a $90 \%$ beam fraction in the range 0.2 to $0.3 \mathrm{~mm}$ are thought to be achievable with gas stripping. The corresponding mass resolution would be 1 part in 11,000 to 16,500 . With a full-width-halfmaximum criterion, the corresponding mass resolution would be 1 part in 16,000 to 24,000 .

- More work is required to estimate achievable beam widths with foil stripping.

\section{DISCUSSION}

Without further modification, the HHIRF tandem accelerator can serve as an excellent RIB accelerator. It's acceptance and minimum injected beam energy requirements are well matched to the sources planned for the ORNL RIB facility; it has good final energy and transmission properties; and it produces highenergy accelerated beams with low emittance and energy spread. With a full-width-half-maximum criterion for mass resolution, the accelerator, as now configured, can provide mass resolutions of the order of 1 part in 6000 for terminal gas stripping and 1 part in 3000 for terminal foil stripping. With the addition of a quadrupole lens upstream of the energyanalyzing magnet, it is believed that the mass resolution with terminal gas stripping could be improved to the order of 1 part in 20,000 . No reduction in beam intensity is required to achieve these resolution values.

The question can be asked as to whether other, smaller tandem accelerators should also be considered for use as radioactive ion beam accelerators. We believe the answer is yes, especially if used in the terminal gas stripping mode. While smaller tandem accelerators will have lower accelerated beam energies, their parameters will, in other respects, be similar or even more favorable than the HHIRF accelerator because acceptance and transmission efficiency will, in general, improve as the length of the acceleration tubes is reduced [6]. The large acceptance and low injection energy requirements of tandem accelerators used in this application have recently assumed greater significance in the light of contributions by Shepard [12] and Talbert [13] who note that viable designs for the first stage of the ISL benchmark radioactive ion beam accelerator [14] capable of accelerating lowvelocily, singly charged, high-mass ions have not yet been developed.

\section{REFERENCES}

[1] "A Proposal for Physics with Exotic Beams at the Holifield Heavy Ion Research Facility," eds. J. D. Garrett and D. K. Olsen (ORNL, February 1991).

[2] "The OR:NL Radioactive Ion Beam Project," D. K. Olsen, et al., Proc. of the Workshop on the Production and Use of Intense Radioactive Beams at the Isospin Laboratory, Oak Ridge, TN, Oct. 7-10, 1992, to be published.

[3] C. M. Jones, et al., Nucl. Instr. and Meth. A268. 308 (1988).

[4] J. K. Bair, et al., IEEE Trans. Nucl. Sci. NS-22, 1655 (1975)

[5] C. M. Jones, Proc. 3rd Int. Conf. on Electrostatic Accelerator Technology, Oak Ridge, TN (Apr. 1981) p. 23.

[6] J. D. Larson and C. M. Jones, Nucl. Instr. and Meth. 140, 489 (1977).

[7] W. T. Milner, et al., IEEE Trans. Nucl. Sci. NS. 22 (3), 1697 (1975).

[8] G. D. Alton and J. W. McConnell, Nuci. instr. and Meth. A268, 445 (1988).

[9] G. D. Alton, et al., Nucl. Instr. and Meth. A328. 325 (1993).

[10] D. L. Haynes, et al., Nucl. Instr. and Meth. A320, 400 (1992).

[11] R. O. Sayer, Revue Phys. Appl. 12, 1543 (1977).

[12] K. W. Shepard, "Some Aspects of Linacs as Applied to the ISL Benchmark Facility," Proc. of the Workshop on the Production and Use of Intense Radioactive Beams at the Isospin Laboratory, Oak Ridge, TN, Oct. 7-10, 1992, to be published.

[13] W. L. Talbert, "Considerations of the LowVelocity Stage of a Radioactive Beams Accelerator," Ibid.

[14] "The IsoSpin Laboratory (ISL) - Research Opportunitics with Radioactive Nuclear Beams," North American Steering Committee for the IsoSpin Laboratory, Los Alamos report LALP 91-51. 

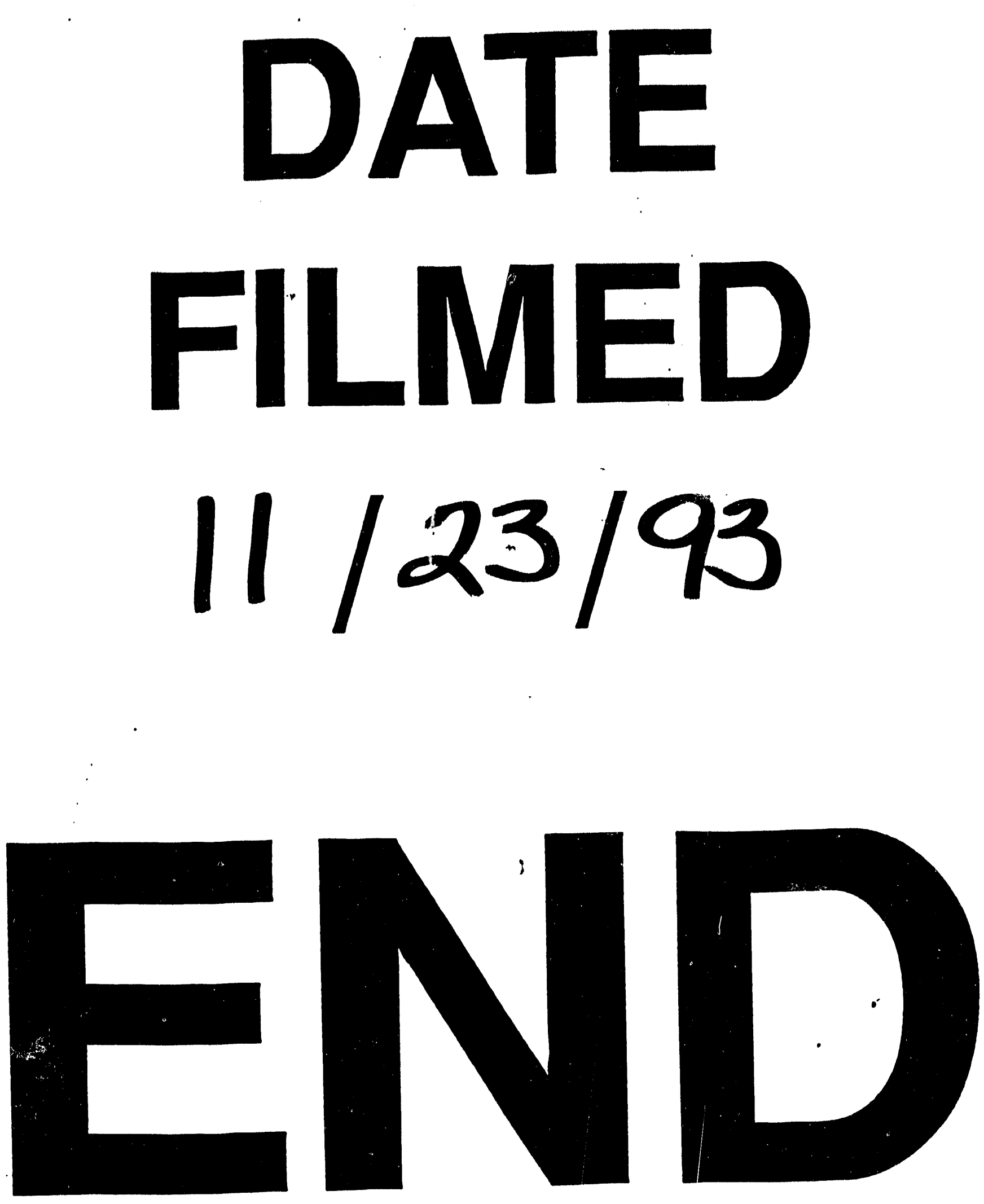
